\title{
Comparison between ray-tracing and full-wave simulation for transcranial ultrasound focusing on a clinical system using the transfer matrix formalism
}

\author{
Thomas Bancel ${ }^{1}$, Alexandre Houdouin ${ }^{1}$, Philippe Annic ${ }^{1}$, Itay Rachmilevitch ${ }^{2}$, Yeruham Shapira ${ }^{2}$, Mickaël Tanter ${ }^{1}$ and Jean- \\ François Aubry ${ }^{1}$.
}

\begin{abstract}
Only one High Intensity Focused Ultrasound device has been clinically approved for transcranial brain surgery at the time of writing. The device operates within $650 \mathrm{kHz}$ and 720 $\mathrm{kHz}$ and corrects the phase distortions induced by the skull of each patient using a multi-element phased array. Phase correction is estimated adaptively using a proprietary algorithm based on computed-tomography (CT) images of the patient's skull. In this paper, we assess the performance of the phase correction computed by the clinical device and compare it to (i) the correction obtained with a previously validated full-wave simulation algorithm using an open-source pseudo-spectral toolbox and (ii) a hydrophone-based correction performed invasively to measure the aberrations induced by the skull at 650 kHz. For the full-wave simulation, three different mappings between CT Hounsfield units and the longitudinal speed of sound inside the skull were tested. All methods are compared with the exact same setup thanks to transfer matrices acquired with the clinical system for $\mathrm{N}=5$ skulls and $\mathrm{T}=2$ different targets for each skull. We show that the clinical ray-tracing software and the full-wave simulation restore respectively $84 \pm 5 \%$ and $86 \pm 5 \%$ of the pressure obtained with hydrophone-based correction for targets located in central brain regions. On the second target (off-center), we also report that the performance of both algorithms degrades when the average incident angles of the acoustic beam at the skull surface increases. When incident angles are higher than $20^{\circ}$, the restored pressure drops below $75 \%$ of the pressure restored with hydrophone-based correction.
\end{abstract}

Index Terms - HIFU, transcranial focusing, ultrasound, numerical modeling, k-wave toolbox, ray-tracing, Insightec, clinical system, aberration correction.

\section{INTRODUCTION}

Transcranial focused ultrasound has been demonstrated to safely ablate deep brain regions involved in neurological diseases, such as essential tremor [1]-[3], Parkinsonian tremor [4] or obsessive compulsive disorders [5]. The main challenge lies in compensating the aberrating effect of the human skull [6], [7] to produce an acoustic beam aiming at the selected target and with a sharp focus [8]. Adaptive corrections are performed by adjusting the phase shift of hundreds of programmable ultrasonic transducers mounted on a hemispherical surface [9], [10].

Extensive research has been conducted for the last 20 years to estimate the phase shifts induced by the skull bone. As reviewed by Kyriakou et al. [11], these techniques range from minimally invasive (implanted hydrophone [12]-[15] or acoustic stars [16], [17]) to non-invasive based on numerical simulations of the ultrasonic propagation through the skull bone with either ray-tracing [10], [18]-[20] angular spectrum [21]-[24], finite difference [25]-[32] or pseudo-spectral schemes [33]-[37]; or a combination of numerical phase estimation adjusted with echoes from injected micro-bubbles [38]. For all numerical methods, the acoustic properties such as density, speed of sound and attenuation are derived from CT or MR imaging of the skull bone [25], [39]-[42].

Most of these numerical approaches were tested either numerically only [19], [23], [28], [30], [31], [35], [43], [44] or with a single-element to assess locally the accuracy of the computed phase shifts [26], [36], [45]-[48]. The improvement of the refocusing with multielement arrays compared to no-correction was assessed experimentally in head phantoms [49]-[51], animal models [52]-[54] or on patients during clinical trials [1]-[5], [38] by measuring the thermal rise at focus with MR thermometry [55], [56] or directly via hydrophone measurements [13], [14], [17], [22], [24], [25], [27], [41], [57]. Quantitative assessment of the performance of the simulation should be performed in terms of percentage of the restored pressure at the target compared to hydrophone-based correction. Hydrophone-based correction uses the phase shifts that are directly measured for all elements with a hydrophone physically located at the target. To our knowledge, only a few publications report the quantitative performance of their numerical models with human skulls positioned in front of a multi-element transducer in terms of pressures restored as compared to a hydrophone-based correction [17], [22], [24], [25], [27], [41], [57], [58] and none of them compared the relative performance of ray-tracing and full-wave simulation approaches on the same experimental setup.

In this article, we assess on the same setup the performance of the two non-invasive trans-skull correction techniques that have been developed historically for implementation in a human device: the proprietary algorithm implemented in the clinical device [59] and a full-wave simulation using k-wave [60] implemented on a transcranial setup [61]. For the fullwave simulation, we used three mappings relating the density to the longitudinal speed of sound inside the skull and previously developed and published by three different labs [39]-[41]. For direct comparison, the transfer matrix formalism was used [61], [62]: a unique transfer matrix was acquired for each skull positioned in front of a clinical 1024element array operated at $650 \mathrm{kHz}$ and used to assess the performance of both simulations. A first target located in the

\footnotetext{
${ }^{1}$ Physics for Medicine Paris, INSERM U1273, ESPCI Paris, CNRS FRE 2031, PSL Research University, Paris, France

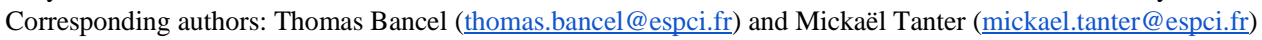

2 InSightec Ltd, 5 Nahum Heth Street, Haifa, Israel.
} 
central part of the brain as well as a second target located $17.5 \mathrm{~mm}$ laterally from the first target and towards the patient's left direction were tested to challenge both techniques and provide insights on how to improve the performance.

\section{MATERIALS AND METHODS}

\section{A. Clinical HIFU system}

Experiments were conducted with an Exablate Neuro 4000 clinical system (Insightec, Tirat Carmel, Israël) installed at the Institut du Cerveau et de la Moëlle Épinière (ICM), located at la Pitié Salpêtrière hospital, Paris. The clinical system is an MR-guided High Intensity Focused Ultrasound (HIFU) system, which has been approved in Europe for thalamotomy [2], [4], [64]-[68]. The ultrasound treatment head is a hemi-spherical transducer with a diameter $\mathrm{D}=300 \mathrm{~mm}$ and is composed of 1024 piezo-electric elements operated at a central frequency $\mathrm{f}_{0}=650 \mathrm{kHz}$.

During clinical treatment, the transducer is used in a vertical position (transducer axis parallel to the ground) and the patient lies on the MR bed with the transducer around its head. In all the experiments described in this article, the transducer was used in a horizontal position (transducer axis perpendicular to the ground) which enabled to fill the transducer with water and to position the skulls inside (Fig. 1.A \& 1.B).

\section{B. Skull preparation}

Five $(\mathrm{N}=5)$ dry skulls were provided by the Institut d'Anatomie (UFR Biomédicale des Saints-Pères, Université Paris Descartes, Paris, France) as approved by the ethics committee of the Centre du Don des Corps (Université Paris Descartes, Paris, France) [61]. The skulls were CT scanned (Sensation Cardiac 64, Siemens, reconstruction kernel H70h) [41], with a voltage of $80 \mathrm{kV}$ and a resolution of $0.45 \times 0.45$ $\mathrm{mm}^{2}$ in the axial plane and a $0.4 \mathrm{~mm}$ slice thickness in the inferior-superior direction.

The skulls were immersed in water prior to each experiment and degassed for at least 12 hours at a 2 mbar reduced pressure (diaphragm pump FB65457, Fisher Scientific, Waltham, MA, USA) to remove the gas trapped in the skull. Each skull was mounted on a stereotactic frame and positioned inside the transducer thanks to a 3D-printed positioning system (Fig. 1.A red box) described in [34] and [37]. The positioning system was then removed and the skull attached to the stereotactic frame was placed inside the transducer previously filled with water (Fig. 1.B).

Two different targets were defined for each skull. In order to compare the results with previously published results [57], the first target was the same as in [57] and was located along the midline of each skull. The second target was located $17.5 \mathrm{~mm}$ away from the first target in the patient's left position (Fig. 1.C). Moving along the left/right axis was motivated by the target used in the treatment of essential tremor [1], and
$17.5 \mathrm{~mm}$ was the maximum distance reachable in order to avoid contact between the screws holding the skulls and the transducer. The stereotactic frame holding the skull was translated so that the target always corresponded to the geometrical center of the transducer.
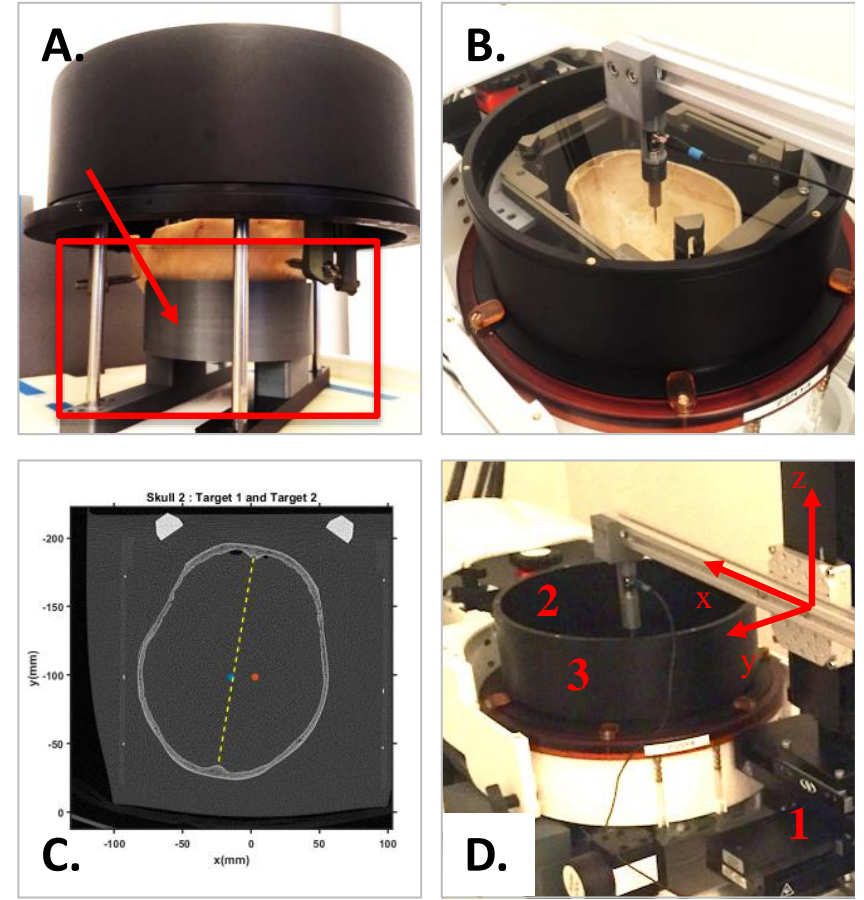

Fig. 1: A: Positioning system (red box) with the 3D-imprint (red arrow) to attach each skull to the stereotactic frame. B: skull positioned inside the transducer after removing the positioning system. C: Axial CT scan for skull \#2 showing the two different targets: first target (blue dot) and second target (orange dot). D: Experimental setup. 1: motorized 3D-positionner (with $x, y$ and $z$ axes), 2: hydrophone holder, 3: Exablate Neuro transducer in horizontal position.

\section{Transfer matrix acquisition}

The transfer matrix was acquired with a needle hydrophone (HNA-0400, ONDA, Sunnyvale, CA, USA, tip dimension: $400 \mu \mathrm{m}$ ) mounted on a motorized 3D positioner (UE41UP, Newport, Irvine, CA, USA) (Fig. 1. B \& D). The hydrophone signal was pre-amplified with an AH-2020 (ONDA, Sunnyvale CA) set to high gain, and further amplified by a 40dB low-noise amplifier (5676 Olympus, Tokyo, Japan). The resulting analog signal was recorded with an oscilloscope (Handyscope HS5, Tiepie, Sneek, Netherlands) at a $100 \mathrm{MHz}$ sampling frequency.

The acoustic focus of the transducer was first determined in a homogeneous medium (without the skull) by finding the position of the maximum pressure field with all the elements of the transducer emitting a spherical wave: 1D (along the $\mathrm{z}$ direction) and 2D (in the $x-y$ plane) scans were iteratively performed, starting from coarse scans $(1 \mathrm{~mm}$ resolution in each direction) to fine scans $(50 \mu \mathrm{m}$ resolution in each direction, corresponding to the spatial resolution of the $3 \mathrm{D}$ positioner).

The water was degassed and cooled before putting the skull in place. Water circulation was stopped before inserting the skull to avoid contamination of the clinical device. The water 
temperature and $\mathrm{O}_{2}$ concentration ranged between $14^{\circ}$ and $22.4^{\circ} \mathrm{C}$ and between 1.2 and $3.9 \mathrm{ppm}$ respectively during the whole set of experiments.

Once each skull was positioned in front of the transducer, a transfer matrix was acquired around the acoustic focus: an ellipsoid volume (small semi-axis: $1 \mathrm{~mm}$, large semi-axis: 1.1 $\mathrm{mm}$ ) was first acquired with a spatial resolution of $0.2 \mathrm{~mm} \mathrm{x}$ $0.2 \mathrm{~mm} \times 0.3 \mathrm{~mm}$, followed by a second ellipsoid volume (small semi-axis: $3 \mathrm{~mm}$, large semi-axis: $4.8 \mathrm{~mm}$ ) with a larger spatial resolution $(0.5 \mathrm{~mm} \times 0.5 \mathrm{~mm} \times 0.8 \mathrm{~mm})$, as displayed in Fig. 2.A. Each scan contained a total of 1345 points. The scanned volume was larger than the focal spot size in water reported by the manufacturer [69]: $1.5 \mathrm{~mm}$ wide in the transverse plane ( $\mathrm{x}-\mathrm{y}$ plane in Fig. 2.A) and $3.0 \mathrm{~mm}$ long in the beam propagation direction (z-axis in Fig. 2.A). The scanned volume inside the transducer is displayed in Figure 2.B. The two different resolutions are visible on Figure 2.A, with a smaller spatial step at the center of the volume (close to the acoustic focus).

A.

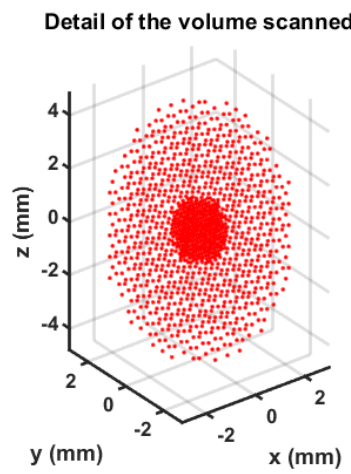

B.

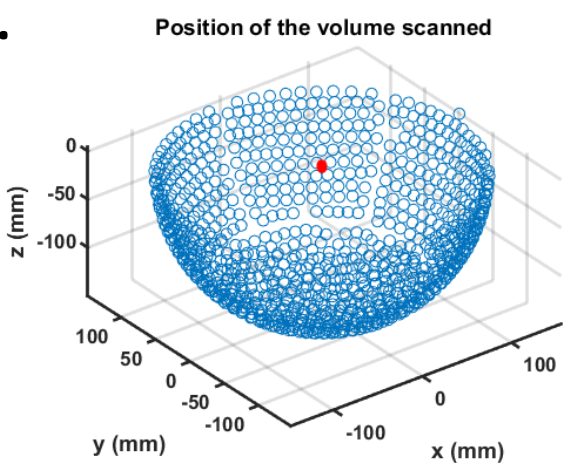

Fig. 2: A: Volume scanned for the transfer matrix acquisition for each skull and each target. B: Position of the volume scanned inside the transducer. The blue circles represent the position of the elements of the transducer.

The elements of the transducer were successively triggered in transmit for each point in space $l \in[1,1345]$, and insonated during $500 \mu \mathrm{s}$ at the same electrical power $(0.5 \mathrm{~W}$ per element). The signal received at the hydrophone was recorded and its amplitude $\left(h_{l i}\right)$ and phase $\left(\varphi_{l i}\right)$ at $650 \mathrm{kHz}$ were extracted for each element $i \in[1,1024]$ of the transducer. The harmonic transfer matrix at $650 \mathrm{kHz}$ was thus recorded for each skull: $H_{l i}(\omega)=h_{l i} . e^{j . \varphi_{l i}}$
The acquisition time for one point in space was approximately 30 seconds: each element insonated for $500 \mu \mathrm{s}$, the system paused for $20 \mathrm{~ms}$ between each of the 1024 element insonation, and we added a 10 s pause before moving to the next point in space to prevent the transducer from heating. The total acquisition time for a 1024 x 1345 transfer matrix was 12 hours for each skull. In total, 10 transfer matrices were acquired (5 skulls x 2 targets) corresponding to more than 10 million measurements.

In addition, hydrophone measurements were performed on 8 points located on a cubic grid ( $1 \mathrm{~mm}$ edge) surrounding the acoustical focus, before and after each transfer matrix acquisition. These hydrophone measurements were used to assess the stability of the setup, as described in the Supplementary Materials.

\section{Pressure field computation with transfer matrix and comparison with direct measurements}

Based on the transfer matrix acquired for each skull and each target, the pressure $\left(p_{l}^{\text {comp }}(\omega)\right)$ generated at position $l$ for a given set of amplitude $a_{i}$ and phase shift $\varphi_{i}$ emitted by element $i$ of the therapy transducer can be computed [41], [57]:

$$
p_{l}^{\text {comp }}(\omega)=\left|\sum_{i=1}^{1024} H_{l i}(\omega) \cdot a_{i} e^{j \cdot \varphi_{l i}}\right|
$$

The pressure fields obtained using the transfer matrix formalism $\left(p^{\text {comp }}\right)$ were compared to the pressure fields measured with the hydrophone when all elements insonate at the same time $\left(p^{\text {meas }}\right)$, with different sets of corrections applied to the transducer.

For each skull, and for the first target only, four different pressure fields $\left(p^{\text {meas }}\right)$ were measured corresponding to four different corrections: no correction (all the elements were in phase), hydrophone-based correction (the phase shift applied on each element corresponded to the negative of the phase shift measured at the focal position), correction provided by the manufacturer's clinical software (Insightec), correction based on the full-wave simulation using Marsac's mapping (more details in the next sections). This resulted in a total of $\mathrm{N}=20$ (5 skulls $\mathrm{x} 4$ corrections) pressure fields scanned with the hydrophone. No amplitude correction was applied for any of the four different corrections: all elements insonated with the same electrical power. The total electrical power across the entire transducer array was $10 \mathrm{~W}$ for each insonation.

The hardware used for signal acquisition was the same as the one described for the acquisition of the transfer matrix except that the low noise amplifier was removed. We programmed the stepper motor to scan the pressure fields at the same location as the acquisition of the transfer matrix points and the insonation time for each point was also $500 \mu \mathrm{s}$.

Measured $\left(p^{\text {meas }}\right)$ and computed $\left(p^{\text {comp }}\right)$ pressure fields were normalized by their maximum value before comparison. For each set of skull and correction, the mean $\left(\hat{p}_{\text {err }}\right)$, standard deviation $\left(\sigma_{e r r}\right)$, root-mean-square $\left(p_{e r r}^{R M S}\right)$ pressure error and the cross-correlation $\left(p^{X C}\right)$ fields were computed as described in [59]: 


$$
\begin{array}{r}
\hat{p}_{\text {err }}=\frac{1}{N} \sum_{i=1}^{N} p_{i}^{\text {comp }}-p_{i}^{\text {meas }} \\
\sigma_{\text {err }}=\sqrt{\frac{1}{N} \sum_{i=1}^{N}\left(p_{i}^{\text {comp }}-p_{i}^{\text {meas }}-\hat{p}_{\text {err }}\right)^{2}} \\
p_{\text {err }}^{\text {RMS }}=\sqrt{\sqrt{\frac{1}{N} \sum_{i=1}^{N}\left(p_{i}^{\text {comp }}-p_{i}^{\text {meas }}\right)^{2}}} \\
p^{X C}=\frac{\sum_{i=1}^{N} p_{i}^{\text {comp }} p_{i}^{\text {meas }}}{\sqrt{\sum_{i=1}^{N}\left(p_{i}^{\text {comp }}\right)^{2} \sum_{i=1}^{N}\left(p_{i}^{\text {meas }}\right)^{2}}}
\end{array}
$$

The shifts between the maximum pressure location of both pressure fields were also computed. When displayed in subsequent figures, pressure fields were interpolated to the resolution of the finest spatial step over 3 (ie. 67 × 67 x 100 $\mu \mathrm{m}^{3}$ ) and centered around the target.

\section{E. Clinical correction}

The CT scans of each skull, as well as the location of the target in the CT coordinates and the position of the transducer with respect to the $\mathrm{CT}$ frame (based on our positioning system) were used as inputs in the manufacturer's clinical software. The phase corrections that would be applied in a clinical treatment were calculated, as well as the incident angle for each element at the skull surface. The average skull density ratio defined for each element as the ratio between Hounsfield Unit (HU) in the marrow layer of the skull bone and the average $\mathrm{HU}$ in the inner and outer cortical bone layers [59] was also calculated by the manufacturer's clinical software.

\section{F. Full-wave simulations}

The propagation of a $500 \mu$ s toneburst of central frequency $\mathrm{f}=650 \mathrm{kHz}$ was simulated through the skull from the target up to each element of the transducer using the k-wave toolbox on Matlab [35], [60]. The full details of the algorithm used in this study is described in [57], we only expose the main principles here.

Briefly, for each skull, acoustic parameters were derived from $\mathrm{HU}$. The k-wave grid was fixed to $760 \mu \mathrm{m}$ to correspond to a grid dimension of $\lambda / 3$ where $\lambda$ is the wavelength in water at $650 \mathrm{kHz}$, to respect the stability criteria of k-wave [35]. The CT was thus first interpolated to fit the spatial resolution of the grid. HU was centered around 1024 and thresholded between 0 and 2400. The thresholded units were noted $\overline{H U}$, and used to compute the skull density using the following linear relationship described in [41]:

$$
\rho=\rho_{\min }+\left(\rho_{\max }-\rho_{\min }\right) \frac{\overline{H U}-\overline{H U}_{\min }}{\overline{H U}_{\min }-\overline{H U}_{\max }}
$$

Values used for $\rho_{\min }\left(\rho_{\min }=1000 \mathrm{~kg} / \mathrm{m}^{3}\right)$ and $\rho_{\max }$ $\left(\rho_{\max }=1900 \mathrm{~kg} / \mathrm{m}^{3}\right)$ were taken from [57]. $\overline{H U}_{\min }$ and
$\overline{H U}_{\text {max }}$ are the minimal and maximal values of $\overline{H U}$ inside the skull.

Then, three different mappings were used to calculate the speed of sound inside the skull. The first one (Marsac's mapping) was based on $\rho$ using the following formula [41]:

$$
c_{\text {Marsac }}=c_{\min }+\left(c_{\max }-c_{\min }\right) \frac{\rho-\rho_{\min }}{\rho_{\min }-\rho_{\max }}
$$

Again, values used for $c_{\min }\left(c_{\min }=1500 \mathrm{~m} / \mathrm{s}\right)$ and $c_{\max }$ $\left(c_{\max }=3100 \mathrm{~m} / \mathrm{s}\right)$ were taken from [57] and $\rho_{\min }$ and $\rho_{\text {max }}$ are minimal and maximal value of the density inside the skull. The second mapping $\left(c_{\text {McDannold }}\right)$ was derived from $\rho$ using McDannold's $4^{\text {th }}$ order polynomial relationship between $c$ and $\rho$ described in [42]. The third mapping $\left(c_{\text {Pichardo }}\right)$ was derived from the multi-frequency characterization of the speed of sound in human skulls by Pichardo et al. (2011) [40]. The speed of sound in the skull bone was linearly interpolated at $650 \mathrm{kHz}$ from spline functions computed from the data presented in Table 9 of [40].

To reduce the computation load, we employed a hybrid-based simulation model [30], [71]. The tone-burst propagation through the skull was simulated using $\mathrm{k}$-wave only from a spherical surface centered on the target and as close as possible to the skull inner surface up to a receiving surface located 5 voxels away from the $500 \overline{\mathrm{HU}}$ iso-surface corresponding to the outer part of the skull (Figure 3) [57], therefore reducing the simulation grid dimensions and the number of timesteps. The propagation in water from this receiving surface to the transducer elements was computed analytically using the second Rayleigh Integral [72].

A.

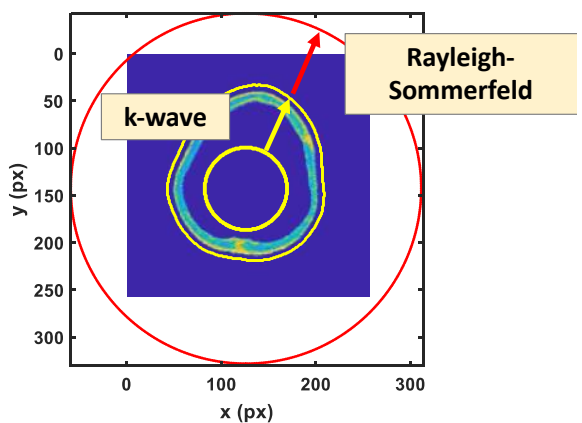

B.

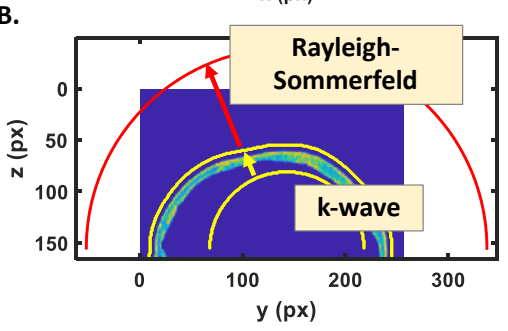

Fig. 3: Propagation from the inner sphere towards the receiving surface (yellow line outside the skull) using $k$-Wave, and from the receiving surface towards the transducer using the second Rayleigh integral. The red and yellow arrows indicate the propagation direction. A: axial view. B: sagittal view.

The temporal time step was automatically calculated by the k-wave toolbox to respect a 0.3 Courant-Friedrich-Lewy (CFL) condition [73] for the most constraining medium, i.e. inside the skull. k-Wave simulations were performed on a 
Titan RTX GPU mounted on an Intel Xeon CPU E5-2630 (v4) operating at $2.2 \mathrm{GHz}$. GPU memory allocation ranged between 6 and $8 \mathrm{~GB}$ for each simulation, depending on the simulation grid size used for each individual skull. The simulation time for one propagation through the skull was $151 \pm 24$ seconds. This simulation time includes $98 \pm 23$ seconds for the propagation through the skull using k-Wave and $13 \pm 1$ seconds for the analytical propagation using the Rayleigh Integral and $40 \pm 4$ seconds for additional processing steps (CT interpolation, extraction of density and speed of sound).

The same algorithm was also tested at three other spatial resolutions: $\lambda / 2, \lambda / 4$ and $\lambda / 5$ where $\lambda$ is the wavelength in water at $650 \mathrm{kHz}$. The results of this convergence test are presented in the Supplementary Materials.

The simulated phase shifts $\left[\varphi_{1}, \ldots, \varphi_{1024}\right]$ on each transducer element were used to either compute the restored pressure in the focal volume using the transfer matrix formalism, or were used as inputs in the clinical system in order to generate the restored pressure field and measure it with the hydrophone (as previously described in section D).

\section{G. Correction performance}

It is known that the aberrations induced by the skull lead to a decrease in the maximum pressure [74], a shifting of the maximum pressure location [41], a spreading of the main lobe [41] and an increase in the sidelobes [74]. Four performance indicators were computed to quantify these effects and assess the performance of the computed corrections:

- the ratio (in \%) of the maximum pressure amplitude divided by the maximum pressure amplitude obtained with hydrophone-based correction;

- the shift of the maximum pressure location with respect to the targeted position (in $\mathrm{mm}$ );

- the increase (in \%) in the $-3 \mathrm{~dB}$ volume compared to the $-3 \mathrm{~dB}$ volume of the hydrophone-based correction;

- $\quad$ the peak sidelobe ratio (PSLR) (in \%) defined as the ratio of the pressure of the largest sidelobe to the pressure of the main lobe.

Pressure fields were computed using the acquired transfer matrices for each set of corrections given by:

- the manufacturer's ray-tracing algorithm

- the full-wave simulation with the three different mappings.

The same analysis was made by isolating the elements based on their incident angles at the surface of the skull. For each skull and target, the transducer elements were split into $k=$ 6 groups (noted $\Omega_{k}$ ) based on their incident angles at the skull surface (provided by the manufacturer's clinical software): for $\mathrm{k}$ ranging from 1 to 5 , each group $\Omega_{k}$ corresponded to an interval of $5^{\circ}\left(\left[0^{\circ}, 5^{\circ}\right]\right.$ to $\left.\left[20^{\circ}, 25^{\circ}\right]\right)$, and $\Omega_{6}$ corresponded to angles beyond $25^{\circ}$. The definition of the incident angle at the skull surface for one element is shown on Figure 4.

For each skull, target and incident angle group $k$, a "subtransfer matrix" $H_{l i}^{k}$ was extracted from the initial transfer matrix. $H_{l i}^{k}$ was restricted to the rows corresponding to transducer elements belonging to $\Omega_{k}$ but included all lines (corresponding to the volumetric points scanned around the acoustic focal):

$$
\mathrm{H}_{\mathrm{li}}^{\mathrm{k}}(\omega)=\mathrm{h}_{\mathrm{li}} \cdot \mathrm{e}^{\mathrm{j} \varphi_{\mathrm{li}}} \text { with } l \in[1,1345] \text { and } i \in \Omega_{k}
$$

For each skull, target and incident angle group $k$, the pressure fields obtained with the manufacturer correction, full-wave simulations (with all mappings) and hydrophone-based correction were calculated by propagating the phase shifts for the selected elements $\varphi_{i}^{\text {manufacturer }}, \varphi_{i}^{\text {full-wave }}$ and $\varphi_{i}^{\text {hydrophone }}$ respectively, using formula (1) for $i \in \Omega_{k}$. The performance of both manufacturer's algorithm and full-wave simulation was assessed as a function of the incident angle interval for each skull and target by comparing the restored pressure fields to the hydrophone-based correction.

A.

\section{Target 1}

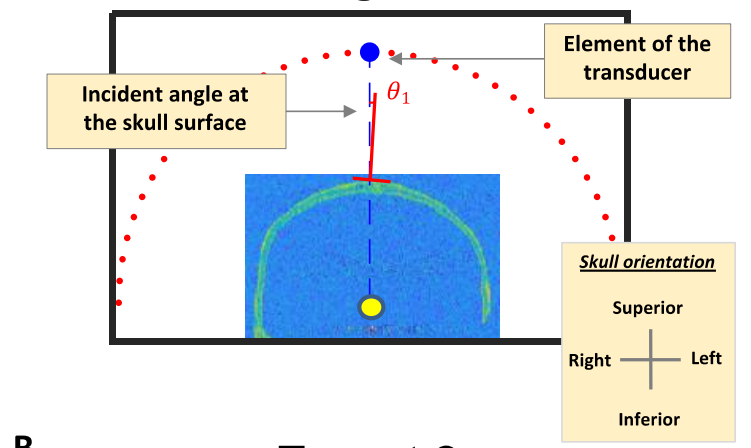

B.

Target 2

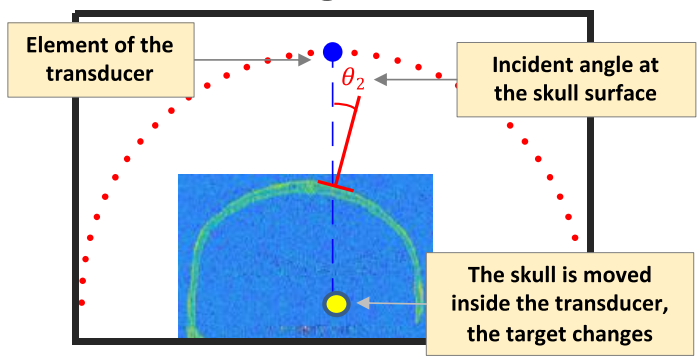

Fig. 4: Incident angle at the skull surface for one element of the transducer. The incident angle changes when the skull is moved inside the transducer between target $1(\boldsymbol{A})$ and target $2(\boldsymbol{B})$.

\section{RESUlts}

For clarity, throughout the entire Results Section, average results are presented along with their standard deviation. We use the notation average \pm standard deviation.

\section{A. Validity of the transfer matrix formalism}

The average difference between measured and computed pressure fields was $-0.9 \pm 5.4 \%\left(\hat{p}_{\text {err }} \pm \sigma_{\text {err }}\right)$. The root mean square error was $5.6 \%\left(p_{e r r}^{R M S}\right)$ and the cross-correlation $\left(p^{X C}\right)$ was $98.9 \%$. The average shift in maximum pressure location between measured and computed pressure fields was $0.3 \pm 0.1 \mathrm{~mm}$. Results are summarized in Table $\mathbf{I}$. 


\begin{tabular}{|c|c|c|c|c|c|}
\hline Skull Number & $\begin{array}{l}\text { Shift of maximum } \\
\text { pressure location }(\mathrm{mm})\end{array}$ & $\hat{p}_{\text {err }}(\%)$ & $\sigma_{\text {err }}(\%)$ & $p_{e r r}^{R M S}(\%)$ & $p^{X C}(\%)$ \\
\hline 1 & 0,3 & $-1,7 \%$ & $6,1 \%$ & $6,6 \%$ & $98,7 \%$ \\
\hline 2 & 0,5 & $-1,1 \%$ & $7,3 \%$ & $7,4 \%$ & $98,0 \%$ \\
\hline 3 & 0,4 & $-0,3 \%$ & $5,4 \%$ & $5,5 \%$ & $98,9 \%$ \\
\hline 4 & 0,1 & $-1,4 \%$ & $4,5 \%$ & $4,7 \%$ & $99,5 \%$ \\
\hline 5 & 0,2 & $-0,2 \%$ & $3,7 \%$ & $3,7 \%$ & $99,4 \%$ \\
\hline Mean & 0,3 , & $-0,9 \%$ & $5,4 \%$ & $5,6 \%$ & $98,9 \%$ \\
\hline
\end{tabular}

Table I : Comparison between computed and measured pressure fields for the first target for 4 corrections (no correction, full-wave simulation with Marsac's mapping, Insightec ray-tracing, hydrophone correction).

Figure 5 displays the shape of the computed $p^{\operatorname{com} p}$ (Fig. 5A) and measured $p^{\text {meas }}$ (Fig. 5B) pressure fields in the focal plane for target 1, skull \#2 without correction. Skull \#2 was chosen to illustrate this study because it is representative of the defocusing effect when no correction is applied. The bottom rows of Figure 5 ( $\mathrm{C}$ to $\mathrm{F}$ ) show the point-by-point comparison of the normalized computed and measured pressure fields in the focal plane, along the coordinates of the target. All the other pressures fields are provided in the supplementary materials.

\section{B. Simulation performance}

The computed phase shifts for each transducer's elements and their corresponding restored pressure fields in the focal plane are displayed in Figure 6 (skull \#2 and first target) for all simulations (manufacturer ray-tracing, full-wave simulation with all three mappings), no correction and hydrophonebased correction.

For both targets, all the corrections were able to restore on average more than $80 \%$ of the pressure obtained with the hydrophone correction. The maximum pressures correction for all skulls and corrections are summarized in Table II. The average performance of the full-wave simulation with Marsac's mapping was $86 \pm 5 \%$ and $84 \pm 4 \%$ for the first and second target respectively. In comparison, the manufacturer's ray-tracing restored $84 \pm 5 \%$ and $83 \pm 6 \%$ of the hydrophonebased correction for the first and second target respectively. Although not statistically significant, the list in increasing average performance is the following: full-wave simulation with Pichardo's mapping, full-wave simulation with McDannold's mapping, ray-tracing from the manufacturer and full-wave simulation with Marsac's mapping.

The decrease in the PSLR followed the same trend. Without correction, the PSLRs were above $60 \%$ for the two targets. The pressure fields obtained after corrections resulted in a reduced PSLR, ranging from $45 \pm 8 \%$ for the full-wave simulation with Marsac's mapping to $52 \pm 11 \%$ for the fullwave with Pichardo's mapping. Hydrophone corrections exhibited a PSLR of 31 and $34 \%$, for the first and the second targets respectively. The standard deviation of the PSLR was also reduced when corrections were applied.

The shift in the maximum pressure location was comprised between 0.2 and $0.3 \mathrm{~mm}$ for all corrections and both targets, corresponding to less than $\lambda / 8$ where $\lambda$ is the wavelength in water at $650 \mathrm{kHz}$, corresponding to an improvement of more than $70 \%$ compared to the shift without correction $(0.8$ and $1.0 \mathrm{~mm}$ for target 1 and 2 respectively). Finally, the $-3 \mathrm{~dB}$ volume ranged from 2.9 to $3.1 \mathrm{~mm}^{3}$. This represents an increase of less than $7 \%$ as compared to the volume at $-3 \mathrm{~dB}$ with hydrophone correction. The results for all targets and corrections in terms of PSLR, shift in maximum pressure location and volume at $-3 \mathrm{~dB}$ are presented in Table III.
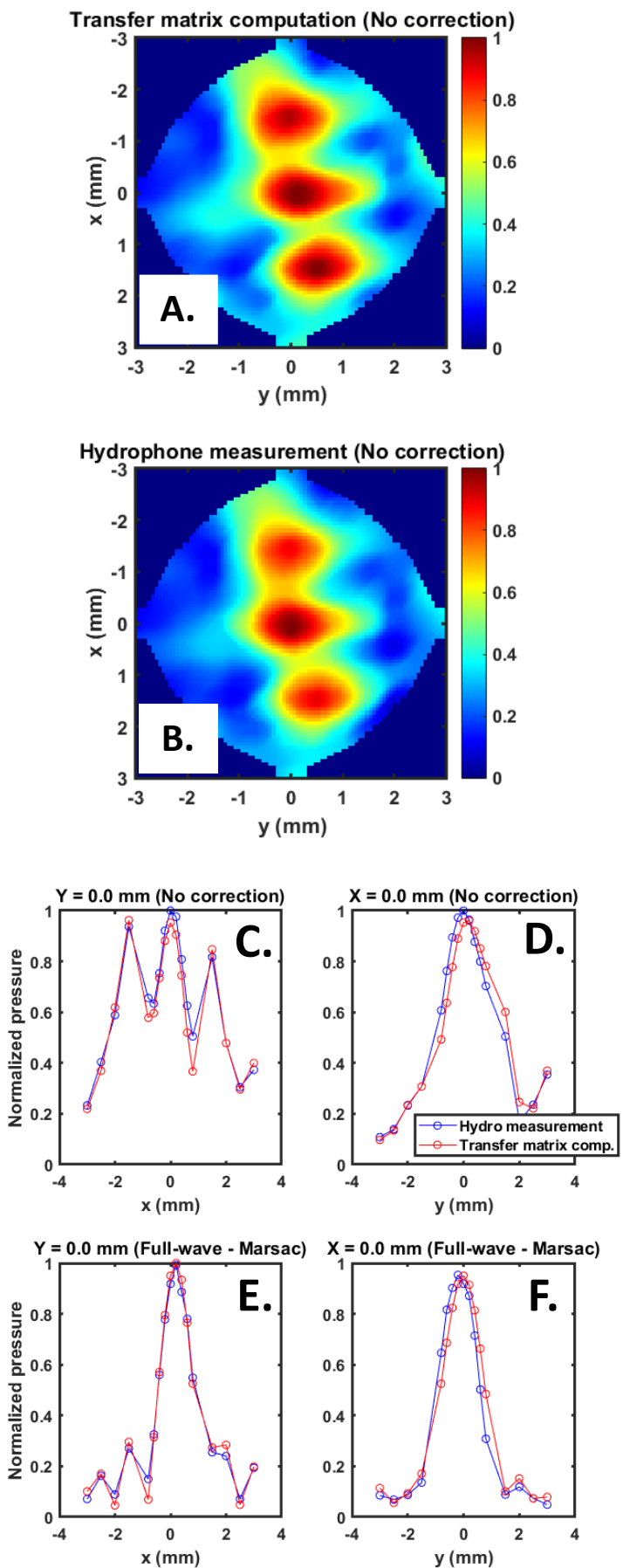

Fig. 5: A \& B: comparison between the pressure fields computed with the transfer matrix formalism $(A)$ and measured with the hydrophone (B) without correction for skull \#2 at the first target. $C$ $\&$ D: point-by-point comparison along the $x$ and $y$-axis without correction. $\boldsymbol{E} \& \boldsymbol{F}$ : point-by-point comparison with the correction from the full-wave simulation (Marsac's mapping). 

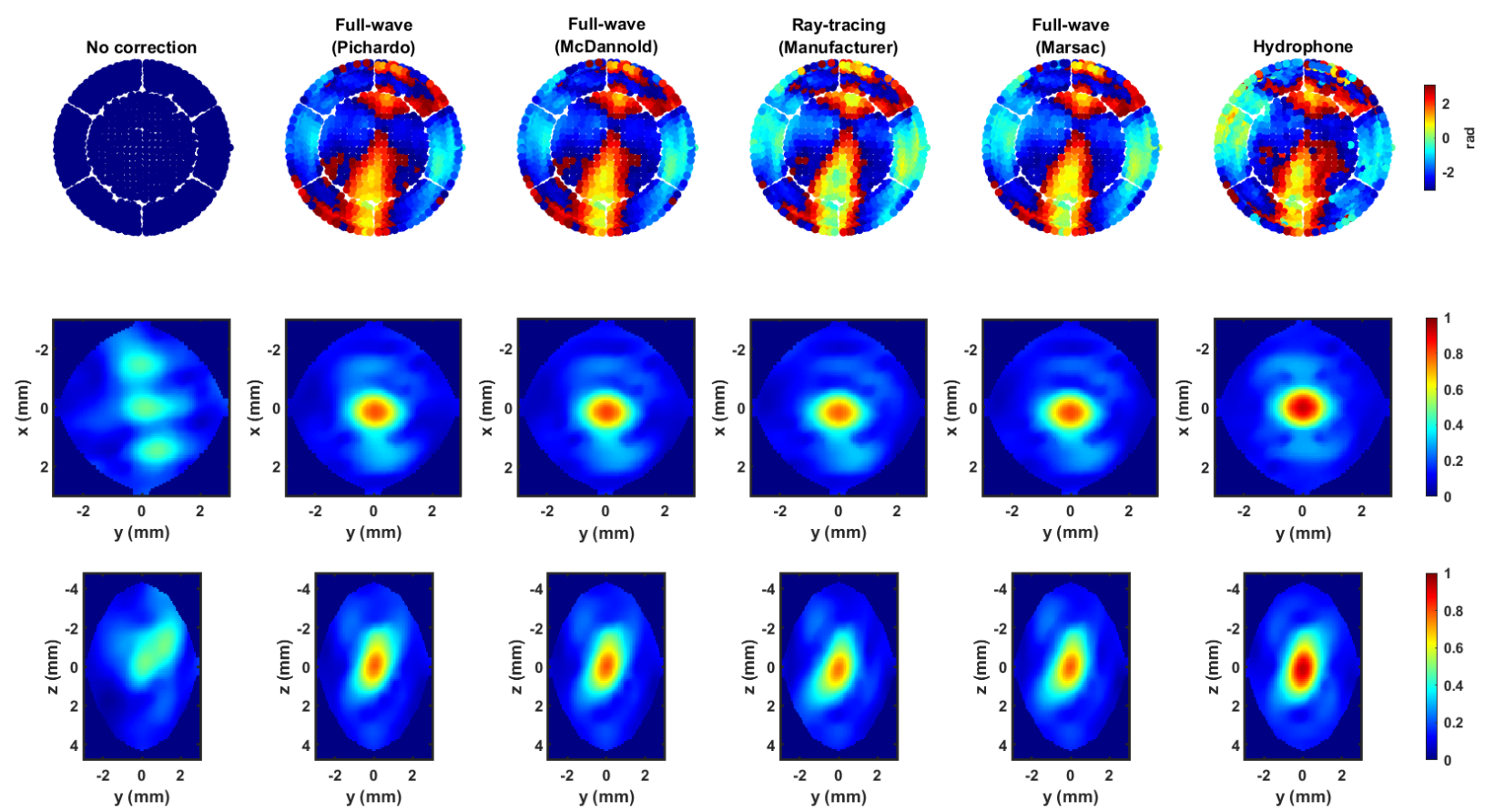

Fig. 6: Pressure fields with different corrections for skull\#2 at the first target. Top row: phase shifts computed by the different algorithms at the surface of the transducer (each dot represents an element of the transducer). Middle row: pressure fields in the axial focal plane $(X-Y$ plane) resulting from the top-row corrections. Bottom row: pressure fields in the $X-Z$ plane $(Z$ is the beam propagation direction).

Target 1

\begin{tabular}{|c|c|c|c|c|c|c|}
\hline Skull Number & $\begin{array}{l}\text { Skull Density } \\
\text { Ratio }\end{array}$ & No correction & $\begin{array}{l}\text { Full-wave } \\
\text { (Pichardo) }\end{array}$ & \begin{tabular}{|l|} 
Full-wave \\
(McDannold)
\end{tabular} & $\begin{array}{l}\text { Manufacturer } \\
\text { correction }\end{array}$ & $\begin{array}{l}\text { Full-wave } \\
\text { (Marsac) }\end{array}$ \\
\hline 1 & 0,37 & $82 \%$ & $90 \%$ & $91 \%$ & $92 \%$ & $92 \%$ \\
\hline 2 & 0,38 & $56 \%$ & $85 \%$ & $87 \%$ & $84 \%$ & $87 \%$ \\
\hline 3 & 0,55 & $61 \%$ & $75 \%$ & $75 \%$ & $78 \%$ & $80 \%$ \\
\hline 4 & 0,57 & $69 \%$ & $81 \%$ & $83 \%$ & $83 \%$ & $86 \%$ \\
\hline 5 & 0,50 & $65 \%$ & $77 \%$ & $79 \%$ & $83 \%$ & $83 \%$ \\
\hline Mean & 0,47 & $67 \%$ & $82 \%$ & $83 \%$ & $84 \%$ & $86 \%$ \\
\hline$S t d$ & 0,09 & $10 \%$ & $6 \%$ & $6 \%$ & $5 \%$ & $5 \%$ \\
\hline
\end{tabular}

Target 2

Skull Number Skull Density No correction \begin{tabular}{l|l|l|l|l} 
& Full-wave & Full-wave & Manufacturer & Full-wave
\end{tabular} \begin{tabular}{crrrrrrr} 
Skull Number & Ratio & No correction & (Pichardo) & (MeDannold) & correction & \multicolumn{2}{c}{ (Marsac) } \\
\hline \hline 1 & 0,37 & $80 \%$ & $90 \%$ & $91 \%$ & $92 \%$ & $91 \%$
\end{tabular} \begin{tabular}{|l|l|l|l|l|l|l|l|}
\hline 2 & 0,38 & $58 \%$ & $83 \%$ & $85 \%$ & $85 \%$ & $86 \%$ \\
\hline
\end{tabular} \begin{tabular}{|l|l|l|l|l|l|l|}
4 & 0,57 & $65 \%$ & $79 \%$ & $80 \%$ & $79 \%$ & $84 \%$ \\
\hline 5 & 0,50 & $60 \%$ & $76 \%$ & $78 \%$ & $82 \%$ & $83 \%$ \\
\hline
\end{tabular}

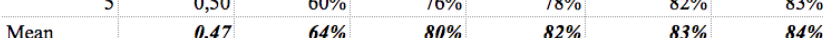

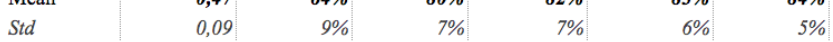

Table II: Performance in terms of restored pressure compared to hydrophone-based correction for each skull for the first (top) and the second (bottom) target.

\begin{tabular}{|c|c|c|c|c|c|c|c|}
\hline & & No correction & $\begin{array}{l}\begin{array}{l}\text { Full-wave } \\
\text { (Pichardo) }\end{array} \\
\end{array}$ & $\begin{array}{l}\begin{array}{l}\text { Full-wave } \\
\text { (McDannold) }\end{array} \\
\end{array}$ & \begin{tabular}{|l|}
$\begin{array}{l}\text { Ray-tracing } \\
\text { (Manufacturer) }\end{array}$ \\
\end{tabular} & $\begin{array}{l}\text { Full-wave } \\
\text { (Marsac) }\end{array}$ & Hydrophone \\
\hline \multirow{3}{*}{ 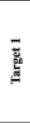 } & $\begin{array}{l}\begin{array}{l}\text { Peak-to-sidelobe Ratio } \\
(\%)\end{array} \\
\text { (1) }\end{array}$ & $63+/-24$ & $52+/-11$ & $51+/-11$ & $46+1-7$ & $45+1-8$ & $31+/-4$ \\
\hline & $\begin{array}{l}\text { Shift in maximum } \\
\text { pressure location (mm) }\end{array}$ & $0.8+/-0.4$ & $0.1+/-0.2$ & $0.1+/-0.2$ & $0.3+/-0.2$ & $0.2+/-0.2$ & 0,00 \\
\hline & $\begin{array}{l}\begin{array}{l}\text { Volume at -3dB } \\
\left(\mathrm{mm}^{\wedge} 3\right)\end{array} \\
\end{array}$ & $3.9+/-0.4$ & $3.1+/-0.2$ & $3.1+/-0.2$ & $3.0+/-0.2$ & $3.1+/-0.1$ & $2.9+/-0.2$ \\
\hline \multirow{3}{*}{ 嚆 } & 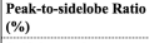 & $69+/-19$ & $51+/-8$ & $52+1-8$ & $48+1-5$ & $46+1-4$ & $34+/-6$ \\
\hline & $\begin{array}{l}\text { Shift in maximum } \\
\text { pressure location (mm) }\end{array}$ & $1.0+/-0.6$ & $0.3+/-0.2$ & $0.3+/-0.2$ & $0.3+/-0.2$ & $0.2+/-0.1$ & 0,00 \\
\hline & $\begin{array}{l}\begin{array}{l}\text { Volume at }-3 \mathrm{~dB} \\
\left(\mathrm{~mm}^{\wedge} 3\right)\end{array}\end{array}$ & $4.0+/-0.5$ & $3.0+/-0.2$ & $3.0+/-0.1$ & $2.9+/-0.2$ & $3.0+/-0.1$ & $2.8+/-0.1$ \\
\hline
\end{tabular}

Table III: PSLR, shift in maximum pressure location and volume at $-3 d B$ averaged over the 5 skulls for each correction and each target.

The full-wave simulation performance was the same when the spatial resolution was reduced to $\lambda / 4$ but the computational time was increased to $459 \pm 75 \mathrm{~s}$. More details are presented in the Supplementary Materials. For comparison, it takes on the order of one second for the manufacturer's algorithm to execute after clicking on the "Calculate" button in the workstation.

\section{Angle distribution performance}

Figure 7.A displays the angle distribution for the two targets. The average incident angle is $8.5 \pm 0.6^{\circ}$ for the first target, it increases to $13.4 \pm 0.6^{\circ}$ for the second target. For the first target (blue bars on Fig. 7.A), less than 50 elements correspond to incident angles above $20^{\circ}$ and only the front part and side parts of the transducer contain elements with angles higher than $15^{\circ}$ (Fig. 7.B). On the contrary, for the second target, all incident angle intervals contain more than 50 elements (orange bars on Fig. 7.A) and elements with incident angles larger than $15^{\circ}$ are located all over the transducer surface (Fig. 7.C).

Fig. 8 displays the average performance of the manufacturer's corrections, the full-wave simulation corrections (for all mappings) and no correction, for each incident angles interval.

Between $0^{\circ}$ and $10^{\circ}$, all corrections perform above $85 \%$ for both targets, whereas for incident angles between $10^{\circ}$ and $20^{\circ}$, the performance drops between $80 \%$ and $85 \%$. For the second target, where more elements have incident angles larger than $20^{\circ}$, the performance of the full-wave simulation with Marsac's mapping and the manufacturer's algorithm continues to drop, with respective values of $76 \pm 15 \%$ and $74 \pm 17 \%$ for angles between 20 and $25^{\circ}$ and of $65 \pm 21 \%$ and $62 \pm 25 \%$ for angles beyond $25^{\circ}$.

The standard deviations of the restored pressure with and without correction increase with incident angles for all corrections. Without correction, for the second target, the standard deviation increases from $6 \%$ for angles below $5^{\circ}$ to $15 \%$ for angles beyond $25^{\circ}$. 
A.
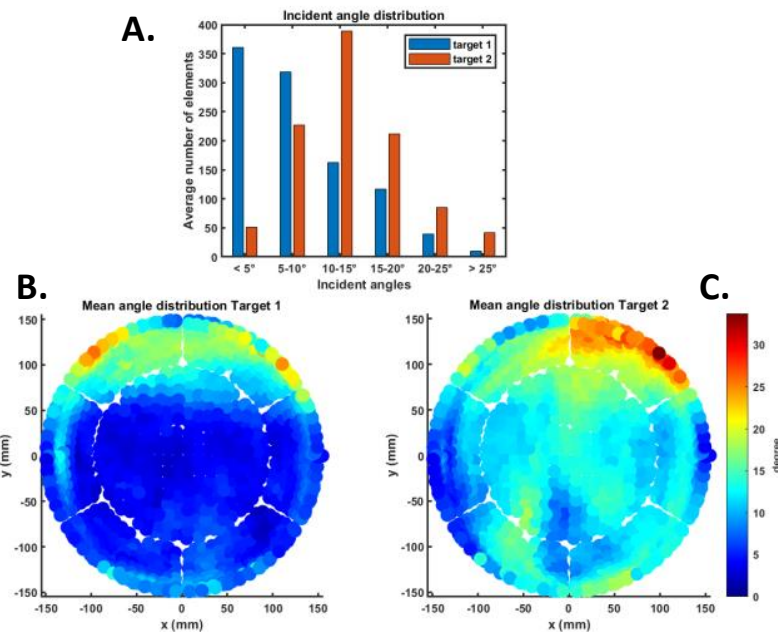

Fig. 7: A: average incident angle distribution for the two targets. $\boldsymbol{B}$ $\& C$ : element incident angle distribution for the transducer's elements for the first $(B)$ and the second $(C)$ target.
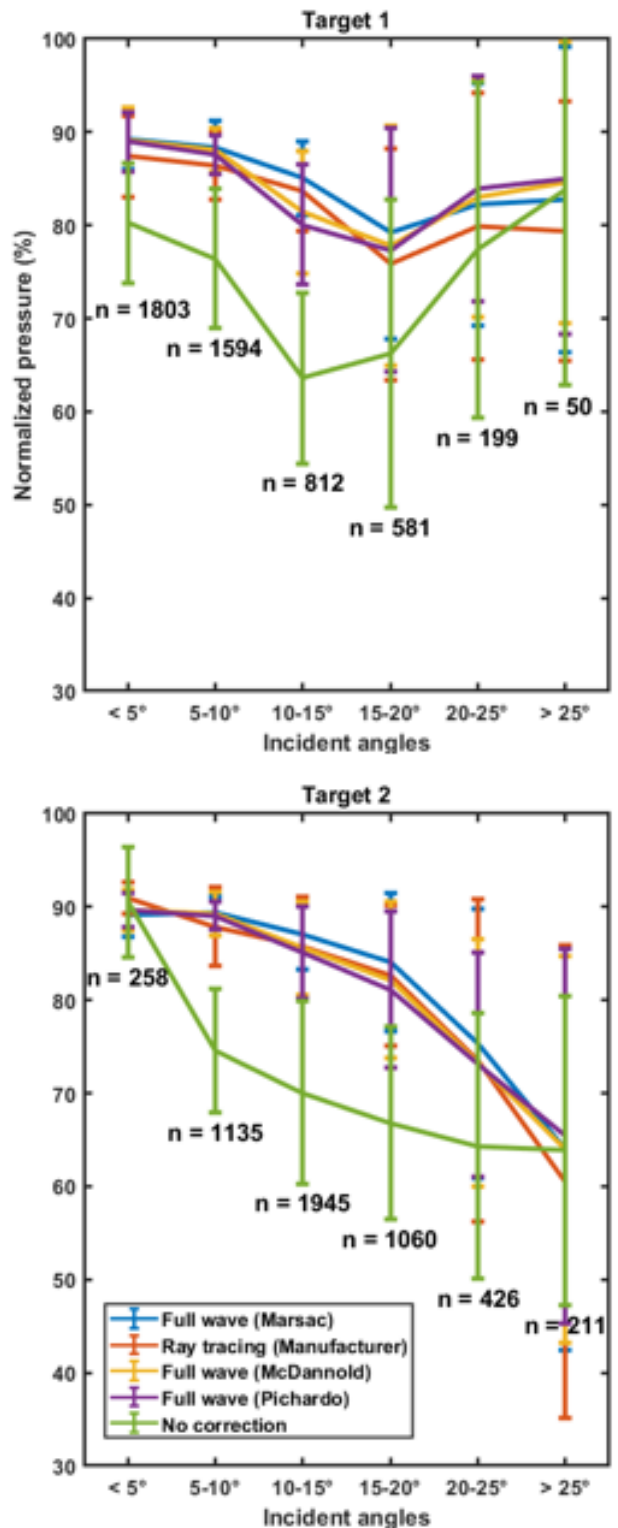

Fig. 8: Algorithm performance based on the incident angle distribution. $n$ represents the number of elements (for the 5 skulls) on which the average performance was computed and the error bars represent the standard deviation.
IV. DISCUSSION

The primary goal of this article was to evaluate whether the use of full-wave transcranial simulation for adaptive aberration corrections would increase the focusing performance compared to ray tracing.

The full-wave simulation presented in this article achieves an average $86 \%$ pressure restoration compared to hydrophonebased correction with Marsac's mapping. To compare our results with results published in [57] using the same skulls, the same target and the same mapping, we compensated the two different transducer geometries by isolating elements from the Exablate-Neuro transducer (blue probe on Fig 9.A) corresponding to the aperture of the pre-clinical transducer used in [57] (orange probe on Fig 9A). The corresponding elements are visible inside the orange circle on Fig 9B. Following the same methodology as for elements with incident angles inside a given interval, we computed the fullwave simulation algorithm performance on those elements and obtained a performance of $89 \pm 4 \%$. Although the impact of changing the operating frequency $(900 \mathrm{kHz}$ in [57] and 650 $\mathrm{kHz}$ in our case) on the performance of full-wave simulations is unknown, such a $89 \pm 4 \%$ performance is in line with the $90 \pm 3 \%$ published in [57].

However, the improvement exhibited in this study in terms of pressure restoration in favor of the full-wave simulation with Marsac's mapping as compared to the ray-tracing correction from the manufacturer appears quite limited. In theory, fullwave simulations should provide a better modelling of the ultrasonic wave propagation in complex environments as compared to ray-tracing approaches, especially when diffraction effects become important and the so-called Eikonal approximation is no more valid [75]. Jones et al. [76] compared the performance of ray-tracing correction and fullwave simulation correction in the field of transcranial passive acoustic imaging. The ray-tracing algorithm modelled the skull as a three layer interface [45] and the full-wave simulation solved the Westervelt equation [77] using the same Pichardo's mapping [40] as the one used in this study. Jones et al. report that the reconstructed images with the fullwave simulation corrections significantly reduced the PSLR, the shift of the detected emission position, the signal-to-noise ratio and the volume at $-3 \mathrm{~dB}$ as compared to images reconstructed with the ray-tracing correction. More recently, Paeng et al. [58] compared the performance of the manufacturer's correction with Kranion, an open-source raytracing algorithm for transcranial aberration correction [18] where the skull is modeled as a single layer. On average, the correction from Insightec improves the restored pressure by more than $10 \%$ as compared to Kranion. Since the Insightec ray-tracing algorithm is proprietary, it is difficult to compare it to other ray-tracing techniques previously published [18], [19], [76], and similarly, comparing different full-wave simulation algorithms [42], [48], [57], [76] without having access to the details of the code is limited. Nevertheless, the manufacturer ray-tracing simulation performed beyond our expectations 
A.

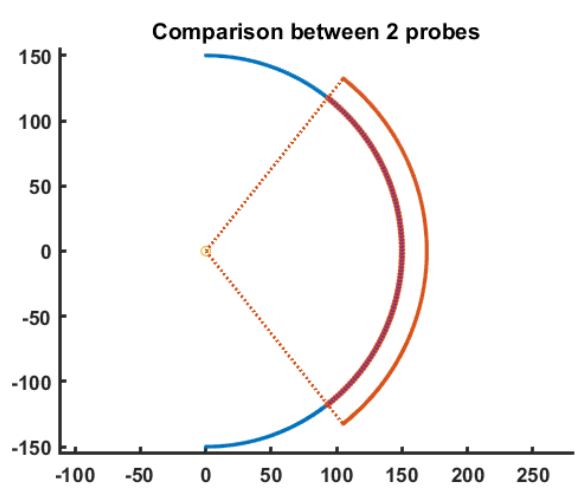

B.

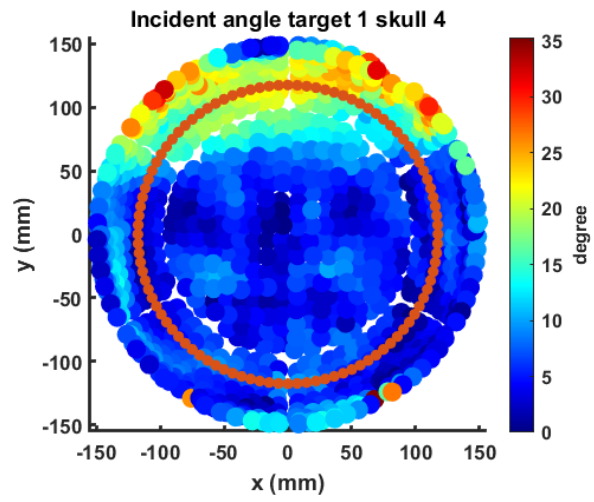

Fig. 9: A: comparison of the geometry of the 512-element preclinical transducer (orange circle) [57] and the clinical (Insightec) transducer used in this study (blue circle). In red overlay, the elements of the clinical transducer facing the same part of the skull as the pre-clinical one. B: incident angles distribution for each element of the clinical transducer. The orange circle shows the elements corresponding to the outer radius of the pre-clinical transducer.

Using a large aperture transducer is optimum for transcranial thermal ablation as it disperses the ultrasound over a large region of the skull [10]. Nevertheless, it also increases the range of incident angles at the outer surface of the skull. Our study shows that the performance of both algorithms tends to degrade for incident angles higher than $20^{\circ}$. It is further confirmed by the increase in standard deviation for incident angles higher than $20^{\circ}$, as compared with no correction (Fig. 8). When incident angles are low, the defocusing variability of each skull, illustrated by the standard deviation of the restored pressure without correction, is compensated by the ability of the algorithms to obtain a better focusing with lower standard deviations. As incident angles increase, the variability between each skull increases (the standard deviation without correction increases) but is no longer compensated by the computed phase corrections. For angles above $25^{\circ}$, algorithms fail to restore more pressure than without correction, and the standard deviation of the corrected pressure is even higher than the standard deviation of the restored pressure without correction.

The SDR (skull density ratio) is a known factor impacting clinical efficiency of HIFU treatments: patients with high SDR exhibit a better temperature rise at the target location [59], [78]. As evidenced by Jung et al. [78], the SDR correlates with the local intensity ratio defined for each element as the amplitude received at the target after passing the skull over the amplitude in water without the skull. This ultimately impacts the performance of HIFU therapy in the clinic, since less energy will pass the skull bone of patients with low SDR. In this article, we performed experiments on $\mathrm{N}=5$ skulls with SDR ranging from 0.37 to 0.57 with a mean value of $0.47 \pm 0.09$. This is comparable to the SDR distribution from Jung et al. However, in our study, the skull density ratio did not impact the performance of either the manufacturer's or the full-wave simulation algorithm. This result supports the hypothesis that low clinical performance for patients with low SDR rather originates from the lack of sufficient energy to heat tissue locally rather than the inability of the algorithm to compensate the dephasing induced by the skull. However, given the high variability of treatment efficiency as a function of SDR [59], this hypothesis would need to be confirmed with a larger number of skulls.

Multiple limitations can degrade the performance of the algorithm. Experimental limitations include the positioning errors of the skull with respect to the transducer, possible errors in the initial localization of the acoustic focus in water with the hydrophone. Simulation limitations include the lack of shear-wave modelling inside the bone, possible inaccurate modeling of the acoustical properties of the skull, or inherent $\mathrm{k}$-wave limitations (band limited interpolation, staircasing [35]). Most of these limitations affect all the ultrasound beams, whereas shear-waves are most likely to take place at high incident angles [45].

It is indeed known that shear-wave propagation plays a significant role for incident angles higher than $20^{\circ}$ [79] and the algorithms tested in this study are based on a fluid model [80], [81] and do not model the longitudinal-transverselongitudinal conversion at the water-skull-brain interface. One possible improvement could be to model shear-wave propagation inside the skull [48], [82], [83] by providing transverse speed maps, and longitudinal and transverse absorption maps inside the skulls [36], [48] to a full-wave simulation algorithm. However, such approach could not be performed in this study because the elastic version of $k$-wave was causing large increases in memory allocation (more than 100GB of RAM was needed for one simulation). Another approach could be to model two rays inside in the skull (one longitudinal and one transverse) recombining inside the brain as a single longitudinal ray as described in [43], [46]. The efficiency of these approaches could be tested with the transfer matrices acquired in this study as all the measurements performed here include the impact of shear waves. Based on performance obtained for low-incident angles, taking into account shear-wave inside the skull could potentially lead to performance above $90 \%$ of pressure restoration compared to hydrophone-based correction. This would be of particularly interest during MRgFUS procedures targeting less centered brain regions [5], [84]. Increased pressures at target from $86 \%$ to $90 \%$ of hydrophone-based correction would result in a $10 \%$ increase in intensity, having a direct impact on treatment efficiency. Additionally, incorporating absorption maps to the full-wave simulation algorithms would also enable to estimate pressure amplitudes, opening room for improvement in amplitude correction during treatment [25], [85].

Modeling transverse propagation inside the skull using a finite difference algorithm would increase the computation time [36], [48]. The spatial sampling interval of the 
simulation scheme must indeed be decreased to respect the stability criteria in the case of shear-wave modeling [35], [36]. Consequently, and as also shown in this study when decreasing the spatial resolution size (Supplementary Materials), computation time increases. Even if such algorithms could in theory be more accurate than the current full-wave simulation, it would require some optimization or increased computation power to be suitable for clinical use. Recently, it has been proposed to decrease the simulation frequency to reduce the simulation grid while maintaining a good level of performance [57]. Such an approach could be extended to shear wave modeling.

It is worth noting here that in this paper all the skulls were immersed in water. During clinical treatments, the ultrasound beams focus through the skin, the skull and various soft tissues (dura, white matter, grey matter, cerebrospinal fluid, etc.). Nevertheless, this is not expected to play a major role compared to the aberrations induced by the skull bone, notwithstanding that the beamformers of current diagnostic ultrasound devices reconstruct the images with a constant speed of sound and neglect the distortions induced by the soft tissue heterogeneities [86].

Finally, this study confirmed that the transfer matrix formalism is a robust methodology to compute pressure fields without the need to scan with the hydrophone for each set of emission signals. It was shown that the pressure field measurements were similar to the calculations using the transfer matrices with a cross-correlation of $99 \%$ and an average difference of $-0.9 \pm 5.4 \%$. The spatial shift of the peak pressure was $0.3 \mathrm{~mm}$, which is comparable to the finest spatial step of the acquisitions $(0.2 \mathrm{~mm} \times 0.2 \mathrm{~mm} \times 0.3 \mathrm{~mm})$. Moreover, the transfer matrix approach allows back-to-back comparison of various sets of simulations. The matrix offers indeed an identical propagation from the transducer array to the measurement points, including the location of the skull, the noise of the electronics, the location of the hydrophone measurements.

\section{CONCLUSIONS}

In this paper, we assessed the performance of the only system currently approved for brain HIFU therapy in terms of (i) percentage of pressure restored compared to hydrophonebased correction, (ii) targeting accuracy, (iii) volume at $-3 \mathrm{~dB}$ and (iv) peak-sidelobe ratio. On average, $84 \%$ of the pressure is restored for central targets and $83 \%$ for $17.5 \mathrm{~mm}$ offcentered targets. A full-wave simulation algorithm was tested as well and showed a slight increase in the performance $(86 \%$ and $84 \%$ for centered and off-centered targets respectively) but with a longer simulation time. Finally, it was shown that the performance was mostly impaired by the higher incident angles, suggesting rooms for improvement with the addition of shear-wave modeling inside the skull bone.

\section{ACKNOWLEDGEMENTS}

This work was achieved in collaboration and with the financial support of Insightec ltd, to which we are grateful. We are also thankful to the Centre de Neuro-Imagerie
(CENIR) of ICM (Institut du Cerveau et de la Moëlle Épinière) Paris, where the experiments were conducted. This work was supported by the Bettencourt Schueller Foundation and the "Agence Nationale de la Recherche" under the program "Future Investments" with the reference ANR-10EQPX-15. The work performed in this publication was performed with the support of the Inserm Technology Research Accelerator (Inserm ART) in Biomedical Ultrasound, Paris, France.

\section{REFERENCES}

[1] W. J. Elias et al., "A Pilot Study of Focused Ultrasound Thalamotomy for Essential Tremor," New England Journal of Medicine, vol. 369, no. 7, pp. 640-648, Aug. 2013, doi: 10.1056/NEJMoa1300962.

[2] N. Lipsman et al., "MR-guided focused ultrasound thalamotomy for essential tremor: a proof-of-concept study," The Lancet Neurology, vol. 12, no. 5, pp. 462-468, May 2013, doi: 10.1016/S1474-4422(13)70048-6.

[3] J. W. Chang et al., "A prospective trial of magnetic resonance-guided focused ultrasound thalamotomy for essential tremor: Results at the 2-year follow-up: 2-Year Follow-up Results of Magnetic Resonance-Guided Focused Ultrasound Thalamotomy for Essential Tremor," Ann Neurol., vol. 83, no. 1, pp. 107-114, Jan. 2018, doi: 10.1002/ana.25126.

[4] R. Martínez-Fernández et al., "Focused ultrasound subthalamotomy in patients with asymmetric Parkinson's disease: a pilot study," The Lancet Neurology, vol. 17, no. 1, pp. 54-63, Jan. 2018, doi: 10.1016/S1474-4422(17)30403-9.

[5] H. H. Jung et al., "Bilateral thermal capsulotomy with MR-guided focused ultrasound for patients with treatmentrefractory obsessive-compulsive disorder: a proof-of-concept study," Mol Psychiatry, vol. 20, no. 10, pp. 1205-1211, Oct. 2015, doi: $10.1038 / \mathrm{mp} .2014 .154$

[6] F. J. Fry and J. E. Barger, "Acoustical properties of the human skull," The Journal of the Acoustical Society of America, vol. 63, no. 5, pp. 1576-1590, May 1978, doi: 10.1121/1.381852.

[7] T. D. Webb et al., "Measurements of the Relationship Between CT Hounsfield Units and Acoustic Velocity and How It Changes With Photon Energy and Reconstruction Method," IEEE Trans. Ultrason., Ferroelect., Freq. Contr., vol. 65, no. 7, pp. 11111124, Jul. 2018, doi: 10.1109/TUFFC.2018.2827899.

[8] J. L. Foley, M. Eames, J. Snell, A. Hananel, N. Kassell, and J.-F. Aubry, "Image-guided focused ultrasound: state of the technology and the challenges that lie ahead," Imaging in Medicine, vol. 5, no. 4, pp. 357-370, Aug. 2013, doi: 10.2217/iim.13.38.

[9] J.-F. Aubry and M. Tanter, "MR-Guided Transcranial Focused Ultrasound," in Therapeutic Ultrasound, vol. 880, J.-M. Escoffre and A. Bouakaz, Eds. Cham: Springer International Publishing, 2016, pp. 97-111.

[10] G. T. Clement, J. White, and K. Hynynen, "Investigation of a large-area phased array for focused ultrasound surgery through the skull," Phys. Med. Biol., vol. 45, no. 4, pp. 1071-1083, Apr. 2000, doi: 10.1088/0031-9155/45/4/319.

[11] A. Kyriakou, E. Neufeld, B. Werner, M. M. Paulides, G. Szekely, and N. Kuster, "A review of numerical and experimental compensation techniques for skull-induced phase aberrations in transcranial focused ultrasound," International Journal of Hyperthermia, vol. 30, no. 1, pp. 36-46, Feb. 2014, doi: 10.3109/02656736.2013.861519.

[12] M. Pernot et al., "In vivo transcranial brain surgery with an ultrasonic time reversal mirror," JNS, vol. 106, no. 6, pp. 10611066, Jun. 2007, doi: 10.3171/jns.2007.106.6.1061.

[13] J.-L. Thomas and M. A. Fink, "Ultrasonic beam focusing through tissue inhomogeneities with a time reversal mirror: application to transskull therapy," IEEE Transactions on Ultrasonics, Ferroelectrics and Frequency Control, vol. 43, no. 6, 
pp. 1122-1129, Nov. 1996, doi: 10.1109/58.542055.

[14] K. Hynynen and F. A. Jolesz, "Demonstration of Potential Noninvasive Ultrasound Brain Therapy Through an Intact Skull," Ultrasound in Medicine \& Biology, vol. 24, no. 2, pp. 275-283, Feb. 1998, doi: 10.1016/S0301-5629(97)00269-X.

[15] G. T. Clement and K. Hynynen, "Micro-receiver guided transcranial beam steering," IEEE Trans. Ultrason., Ferroelect., Freq. Contr., vol. 49, no. 4, pp. 447-453, Apr. 2002, doi: $10.1109 / 58.996562$.

[16] M. Pernot, G. Montaldo, M. Tanter, and M. Fink, "'Ultrasonic stars' for time reversal focusing using induced cavitation bubbles.," in Therapeutic Ultrasound: ISTU, 2006, p. 6. [17] J. Gateau, L. Marsac, M. Pernot, J.-F. Aubry, M. Tanter, and M. Fink, "Transcranial Ultrasonic Therapy Based on Time Reversal of Acoustically Induced Cavitation Bubble Signature," IEEE Trans. Biomed. Eng., vol. 57, no. 1, pp. 134-144, Jan. 2010, doi: 10.1109/TBME.2009.2031816.

[18] F. Sammartino, D. W. Beam, J. Snell, and V. Krishna, "Kranion, an open-source environment for planning transcranial focused ultrasound surgery: technical note," Journal of Neurosurgery, vol. 132, pp. 987-1311, Apr. 2020, doi: https://doi.org/10.3171/2018.11.JNS181995.

[19] J. Sun and K. Hynynen, "Focusing of therapeutic ultrasound through a human skull: A numerical study," The Journal of the Acoustical Society of America, vol. 104, no. 3, pp. 1705-1715, Sep. 1998, doi: 10.1121/1.424383.

[20] D. Pajek and K. Hynynen, "The design of a focused ultrasound transducer array for the treatment of stroke: a simulation study," Phys. Med. Biol., vol. 57, no. 15, pp. 4951-4968, Aug. 2012, doi: 10.1088/0031-9155/57/15/4951.

[21] S. A. Leung, T. D. Webb, R. R. Bitton, P. Ghanouni, and K. Butts Pauly, "A rapid beam simulation framework for transcranial focused ultrasound,"Sci Rep, vol. 9, no. 1, p. 7965, Dec. 2019, doi: 10.1038/s41598-019-43775-6.

[22] S. Almquist, D. L. Parker, and D. A. Christensen, "Rapid full-wave phase aberration correction method for transcranial highintensity focused ultrasound therapies," J Ther Ultrasound, vol. 4, no. 1, p. 30, Dec. 2016, doi: 10.1186/s40349-016-0074-7.

[23] S. Schoen and C. D. Arvanitis, "Heterogeneous Angular Spectrum Method for Trans-Skull Imaging and Focusing," IEEE Trans. Med. Imaging, vol. 39, no. 5, pp. 1605-1614, May 2020, doi: 10.1109/TMI.2019.2953872.

[24] G. T. Clement and K. Hynynen, "A non-invasive method for focusing ultrasound through the human skull," Phys. Med. Biol., vol. 47, no. 8, pp. 1219-1236, Apr. 2002, doi: 10.1088/0031$9155 / 47 / 8 / 301$

[25] J.-F. Aubry, M. Tanter, M. Pernot, J.-L. Thomas, and M. Fink, "Experimental demonstration of noninvasive transskull adaptive focusing based on prior computed tomography scans," The Journal of the Acoustical Society of America, vol. 113, no. 1, pp. 8493, Jan. 2003, doi: 10.1121/1.1529663.

[26] G. Pinton, J.-F. Aubry, E. Bossy, M. Muller, M. Pernot, and M. Tanter, "Attenuation, scattering, and absorption of ultrasound in the skull bone: Absorption of ultrasound in the skull bone," Med. Phys., vol. 39, no. 1, pp. 299-307, Dec. 2011, doi: 10.1118/1.3668316.

[27] F. Marquet et al., "Non-invasive transcranial ultrasound therapy based on a 3D CT scan: protocol validation and in vitro results," Physics in Medicine and Biology, vol. 54, no. 9, pp. 2597 2613, May 2009, doi: 10.1088/0031-9155/54/9/001.

[28] G. Pinton, J.-F. Aubry, M. Fink, and M. Tanter, "Numerical prediction of frequency dependent 3D maps of mechanical index thresholds in ultrasonic brain therapy: Mechanical index thresholds in ultrasonic brain therapy," Med. Phys., vol. 39, no. 1, pp. 455-467, Dec. 2011, doi: 10.1118/1.3670376.

[29] G. Pinton, J.-F. Aubry, M. Fink, and M. Tanter, "Effects of nonlinear ultrasound propagation on high intensity brain therapy: Nonlinear ultrasound propagation and high intensity brain therapy," Med. Phys., vol. 38, no. 3, pp. 1207-1216, Feb. 2011, doi: 10.1118/1.3531553.

[30] G. Pinton, J.-F. Aubry, and M. Tanter, "Direct phase projection and transcranial focusing of ultrasound for brain therapy," IEEE Trans. Ultrason., Ferroelect., Freq. Contr., vol. 59, no. 6, pp. 1149-1159, Jun. 2012, doi: 10.1109/TUFFC.2012.2305.

[31] C. Baron, J.-F. Aubry, M. Tanter, S. Meairs, and M. Fink, "Simulation of Intracranial Acoustic Fields in Clinical Trials of Sonothrombolysis," Ultrasound in Medicine \& Biology, vol. 35, no. 7, pp. 1148-1158, Jul. 2009, doi: 10.1016/j.ultrasmedbio.2008.11.014.

[32] G. Bouchoux et al., "Experimental validation of a finitedifference model for the prediction of transcranial ultrasound fields based on CT images," Phys. Med. Biol., vol. 57, no. 23, pp. 80058022, Dec. 2012, doi: 10.1088/0031-9155/57/23/8005.

[33] Y. Jing, F. C. Meral, and G. T. Clement, "Time-reversal transcranial ultrasound beam focusing using a k-space method," Phys. Med. Biol., vol. 57, no. 4, pp. 901-917, Feb. 2012, doi: 10.1088/0031-9155/57/4/901.

[34] G. Maimbourg, A. Houdouin, T. Deffieux, M. Tanter, and J.-F. Aubry, "3D-printed adaptive acoustic lens as a disruptive technology for transcranial ultrasound therapy using single-element transducers," Physics in Medicine \& Biology, vol. 63, no. 2, p. 025026, Jan. 2018, doi: 10.1088/1361-6560/aaa037.

[35] J. L. B. Robertson, B. T. Cox, J. Jaros, and B. E. Treeby, "Accurate simulation of transcranial ultrasound propagation for ultrasonic neuromodulation and stimulation," J. Acoust. Soc. Am., vol. 141, no. 3, pp. 1726-1738, Mar. 2017, doi: 10.1121/1.4976339. [36] J. Robertson, E. Martin, B. Cox, and B. E. Treeby, "Sensitivity of simulated transcranial ultrasound fields to acoustic medium property maps," Phys. Med. Biol., vol. 62, no. 7, pp. 2559 2580, Apr. 2017, doi: 10.1088/1361-6560/aa5e98.

[37] G. Maimbourg, A. Houdouin, T. Deffieux, M. Tanter, and J.-F. Aubry, "Steering Capabilities of an Acoustic Lens for Transcranial Therapy: Numerical and Experimental Studies," IEEE Trans. Biomed. Eng., vol. 67, no. 1, pp. 27-37, Jan. 2020, doi: 10.1109/TBME.2019.2907556.

[38] R. M. Jones et al., "Echo-Focusing in Transcranial Focused Ultrasound Thalamotomy for Essential Tremor: A Feasibility Study," Mov Disord, vol. 35, no. 12, pp. 2327-2333, Dec. 2020, doi: $10.1002 / \mathrm{mds} .28226$.

[39] C. W. Connor, G. T. Clement, and K. Hynynen, "A unified model for the speed of sound in cranial bone based on genetic algorithm optimization," Phys. Med. Biol., vol. 47, no. 22, pp. 39253944, Nov. 2002, doi: 10.1088/0031-9155/47/22/302.

[40] S. Pichardo, V. W. Sin, and K. Hynynen, "Multifrequency characterization of the speed of sound and attenuation coefficient for longitudinal transmission of freshly excised human skulls," Phys. Med. Biol., vol. 56, no. 1, pp. 219-250, Jan. 2011, doi: 10.1088/0031-9155/56/1/014.

[41] L. Marsac et al. "Ex vivo optimisation of a heterogeneous speed of sound model of the human skull for non-invasive transcranial focused ultrasound at $1 \mathrm{MHz}$," International Journal of Hyperthermia, vol. 33, no. 6, pp. 635-645, Aug. 2017, doi: 10.1080/02656736.2017.1295322.

[42] N. McDannold, P. J. White, and R. Cosgrove, "Elementwise approach for simulating transcranial MRI-guided focused ultrasound thermal ablation," Phys. Rev. Research, vol. 1, no. 3, p. 033205, Dec. 2019, doi: 10.1103/PhysRevResearch.1.033205.

[43] S. Pichardo and K. Hynynen, "Treatment of near-skull brain tissue with a focused device using shear-mode conversion: a numerical study," Phys. Med. Biol., vol. 52, no. 24, pp. 7313-7332, Dec. 2007, doi: 10.1088/0031-9155/52/24/008.

[44] U. Vyas and D. Christensen, "Ultrasound beam simulations in inhomogeneous tissue geometries using the hybrid angular spectrum method," IEEE Trans. Ultrason., Ferroelect., Freq. Contr., vol. 59, no. 6, pp. 1093-1100, Jun. 2012, doi: 10.1109/TUFFC.2012.2300.

[45] G. T. Clement and K. Hynynen, "Correlation of ultrasound phase with physical skull properties," Ultrasound in Medicine \& Biology, vol. 28, no. 5, pp. 617-624, May 2002, doi: 10.1016/S03015629(02)00503-3.

[46] G. T. Clement, P. J. White, and K. Hynynen, "Enhanced 
ultrasound transmission through the human skull using shear mode conversion," The Journal of the Acoustical Society of America, vol. 115, no. 3, pp. 1356-1364, Mar. 2004, doi: 10.1121/1.1645610.

[47] J. Aarnio, G. T. Clement, and K. Hynynen, "A new ultrasound method for determining the acoustic phase shifts caused by the skull bone," Ultrasound in Medicine \& Biology, vol. 31, no. 6, pp. 771-780, Jun. 2005, doi: 10.1016/j.ultrasmedbio.2005.01.019.

[48] S. Pichardo, C. Moreno-Hernández, R. Andrew Drainville, V. Sin, L. Curiel, and K. Hynynen, "A viscoelastic model for the prediction of transcranial ultrasound propagation: application for the estimation of shear acoustic properties in the human skull," Phys. Med. Biol., vol. 62, no. 17, pp. 6938-6962, Aug. 2017, doi: 10.1088/1361-6560/aa7ccc.

[49] M. D. C. Eames et al., "Head phantoms for transcranial focused ultrasound: Head phantoms for transcranial focused ultrasound," Med. Phys., vol. 42, no. 4, pp. 1518-1527, Mar. 2015, doi: 10.1118/1.4907959.

[50] M. Wintermark et al., "T1-weighted MRI as a substitute to CT for refocusing planning in MR-guided focused ultrasound," Phys. Med. Biol., vol. 59, no. 13, pp. 3599-3614, Jul. 2014, doi: 10.1088/0031-9155/59/13/3599.

[51] G. W. Miller, M. Eames, J. Snell, and J.-F. Aubry, "Ultrashort echo-time MRI versus CT for skull aberration correction in MR-guided transcranial focused ultrasound: In vitro comparison on human calvaria: UTE-based skull aberration correction for MRguided HIFU," Med. Phys., vol. 42, no. 5, pp. 2223-2233, Apr. 2015, doi: 10.1118/1.4916656.

[52] N. McDannold et al., "MRI-guided focused ultrasound surgery in the brain: Tests in a primate model," Magn. Reson. Med., vol. 49, no. 6, pp. 1188-1191, Jun. 2003, doi: 10.1002/mrm.10453. [53] W. J. Elias et al., "A magnetic resonance imaging, histological, and dose modeling comparison of focused ultrasound, radiofrequency, and Gamma Knife radiosurgery lesions in swine thalamus," JNS, vol. 119, no. 2, pp. 307-317, Aug. 2013, doi: 10.3171/2013.5.JNS122327.

[54] K. Hynynen et al., "Pre-clinical testing of a phased array ultrasound system for MRI-guided noninvasive surgery of the brain-A primate study," European Journal of Radiology, vol. 59, no. 2, pp. 149-156, Aug. 2006, doi: 10.1016/j.ejrad.2006.04.007.

[55] V. Rieke et al., "Comparison of temperature processing methods for monitoring focused ultrasound ablation in the brain: Temperature Processing Methods," J. Magn. Reson. Imaging, vol. 38, no. 6, pp. 1462-1471, Dec. 2013, doi: 10.1002/jmri.24117.

[56] V. Ozenne et al., "MRI monitoring of temperature and displacement for transcranial focus ultrasound applications," NeuroImage, vol. 204, p. 116236, Jan. 2020, doi: 10.1016/j.neuroimage.2019.116236.

[57] G. Maimbourg et al., "Computationally efficient transcranial ultrasonic focusing: taking advantage of the high correlation length of the human skull," IEEE Transactions on Ultrasonics, Ferroelectrics and Frequency Control, vol. 67, no. 10, pp. 1993-2002, May 2020.

[58] C. Jin, D. Moore, J. Snell, and D.-G. Paeng, "An opensource phase correction toolkit for transcranial focused ultrasound," BMC biomed eng, vol. 2, no. 1, p. 9, Dec. 2020, doi: 10.1186/s42490-020-00043-3.

[59] W. S. Chang et al., "Factors associated with successful magnetic resonance-guided focused ultrasound treatment: efficiency of acoustic energy delivery through the skull," Journal of Neurosurgery, vol. 124, no. 2, pp. 411-416, Feb. 2016, doi: 10.3171/2015.3.JNS142592.

[60] A. B. Treeby, B. Cox, and J. Jaros, "KWAVE: A MATLAB toolbox for the time domain simulation of acoustic wave fields User Manual,” p. 88, 2016.

[61] D. Chauvet et al., "Targeting accuracy of transcranial magnetic resonance-guided high-intensity focused ultrasound brain therapy: a fresh cadaver model," JNS, vol. 118, no. 5, pp. 10461052, May 2013, doi: 10.3171/2013.1.JNS12559.

[62] M. Tanter, J.-F. Aubry, J. Gerber, J.-L. Thomas, and M. Fink, "Optimal focusing by spatio-temporal inverse filter. I. Basic principles," The Journal of the Acoustical Society of America, vol. 110, no. 1, pp. 37-47, Jul. 2001, doi: 10.1121/1.1377051.

[63] J.-F. Aubry, M. Tanter, J. Gerber, J.-L. Thomas, and M. Fink, "Optimal focusing by spatio-temporal inverse filter. II. Experiments. Application to focusing through absorbing and reverberating media," The Journal of the Acoustical Society of America, vol. 110, no. 1, pp. 48-58, Jul. 2001, doi: 10.1121/1.1377052.

[64] D. Jeanmonod et al., "Transcranial magnetic resonance imaging-guided focused ultrasound: noninvasive central lateral thalamotomy for chronic neuropathic pain," FOC, vol. 32, no. 1, p. E1, Jan. 2012, doi: 10.3171/2011.10.FOCUS11248.

[65] A. Magara, R. Bühler, D. Moser, M. Kowalski, P. Pourtehrani, and D. Jeanmonod, "First experience with MR-guided focused ultrasound in the treatment of Parkinson's disease," J Ther Ultrasound, vol. 2, no. 1, p. 11, 2014, doi: 10.1186/2050-5736-2-11. [66] J. U. Máñez-Miró et al., "Focused ultrasound thalamotomy for multiple sclerosis-associated tremor," Mult Scler, p. 135245851986159 , Oct. 2019, doi: 10.1177/1352458519861597. [67] L. Bretsztajn and W. Gedroyc, "Brain-focussed ultrasound: what's the 'FUS' all about? A review of current and emerging neurological applications," BJR, p. 20170481, Mar. 2018, doi: 10.1259/bjr.20170481

[68] W. J. Elias et al., "A Randomized Trial of Focused Ultrasound Thalamotomy for Essential Tremor," N Engl J Med, vol. 375, no. 8, pp. 730-739, Aug. 2016, doi: 10.1056/NEJMoa1600159. [69] Inc. Insightec, "Exablate 4000 Type 1.1 SW 7.2 Operator Manual CE Rev5.8.” Jan. 2019.

[70] R. Xu and M. A. O'Reilly, "Simulating transvertebral ultrasound propagation with a multi-layered ray acoustics model," Phys. Med. Biol., vol. 63, no. 14, p. 145017, Jul. 2018, doi: 10.1088/1361-6560/aacf75.

[71] A. Pulkkinen, Y. Huang, J. Song, and K. Hynynen, "Simulations and measurements of transcranial low-frequency ultrasound therapy: skull-base heating and effective area of treatment," Phys. Med. Biol., vol. 56, no. 15, pp. 4661-4683, Aug. 2011, doi: 10.1088/0031-9155/56/15/003

[72] D. Royer and E. Dieulesaint, Elastic Waves in Solids I: Free and Guided Propagation, Springer. 1999.

[73] R. Courant, K. Friedrichs, and H. Lewy, On the partial difference equations of Mathematical Physics. 1956.

[74] D. N. White, J. M. Clark, J. N. Chesebrough, M. N. White, and J. K. Campbell, "Effect of the Skull in Degrading the Display of Echoencephalographic B and C Scans," J. Acoust. Soc. Am., vol. 44, pp. 1339-1345, 1968.

[75] R. Prislan, G. Veble, and D. Svenšek, "Ray-trace modeling of acoustic Green's function based on the semiclassical (eikonal) approximation," The Journal of the Acoustical Society of America, vol. 140, no. 4, pp. 2695-2702, Oct. 2016, doi: 10.1121/1.4964295.

[76] R. M. Jones and K. Hynynen, "Comparison of analytical and numerical approaches for CT-based aberration correction in transcranial passive acoustic imaging," Phys. Med. Biol., vol. 61, no. 1, pp. 23-36, Jan. 2016, doi: 10.1088/0031-9155/61/1/23.

[77] P. J. Westervelt, "Parametric Acoustic Array," The Journal of the Acoustical Society of America, vol. 35, no. 4, pp. 535537, Apr. 1963, doi: 10.1121/1.1918525.

[78] N. Y. Jung, I. Rachmilevitch, O. Sibiger, T. Amar, E. Zadicario, and J. W. Chang, "Factors Related to Successful Energy Transmission of Focused Ultrasound through a Skull : A Study in Human Cadavers and Its Comparison with Clinical Experiences," $J$ Korean Neurosurg Soc, May 2019, doi: 10.3340/jkns.2018.0226.

[79] M. Hayner and K. Hynynen, "Numerical analysis of ultrasonic transmission and absorption of oblique plane waves through the human skull," The Journal of the Acoustical Society of America, vol. 110, no. 6, pp. 3319-3330, Dec. 2001, doi: 10.1121/1.1410964

[80] J. Jaros, A. P. Rendell, and B. E. Treeby, "Full-wave nonlinear ultrasound simulation on distributed clusters with applications in high-intensity focused ultrasound," The International Journal of High Performance Computing 
Applications, vol. 30, no. 2, pp. 137-155, May 2016, doi: $10.1177 / 1094342015581024$

[81] B. E. Treeby, J. Jaros, A. P. Rendell, and B. T. Cox, "Modeling nonlinear ultrasound propagation in heterogeneous media with power law absorption using a k-space pseudospectral method," The Journal of the Acoustical Society of America, vol. 131, no. 6, pp. 4324-4336, Jun. 2012, doi: 10.1121/1.4712021.

[82] A. Pulkkinen, B. Werner, E. Martin, and K. Hynynen, "Numerical simulations of clinical focused ultrasound functional neurosurgery," Phys. Med. Biol., vol. 59, no. 7, pp. 1679-1700, Apr. 2014, doi: 10.1088/0031-9155/59/7/1679.

[83] Junho Song, A. Pulkkinen, Yuexi Huang, and K. Hynynen, "Investigation of Standing-Wave Formation in a Human Skull for a Clinical Prototype of a Large-Aperture, Transcranial MR-Guided Focused Ultrasound (MRgFUS) Phased Array: An Experimental and Simulation Study," IEEE Trans. Biomed. Eng., vol. 59, no. 2, pp. 435-444, Feb. 2012, doi:
10.1109/TBME.2011.2174057.

[84] W. E. Parker et al., "Magnetic resonance-guided focused ultrasound for ablation of mesial temporal epilepsy circuits: modeling and theoretical feasibility of a novel noninvasive approach," Journal of Neurosurgery, vol. 133, no. 1, pp. 63-70, Jul. 2020, doi: 10.3171/2019.4.JNS182694.

[85] J. White, G. T. Clement, and K. Hynynen, "Transcranial Ultrasound Focus Reconstruction with Phase and Amplitude Correction," vol. 52, no. 9, p. 5, 2005.

[86] T. Szabo L., Diagnostic Ultrasound Imaging: Inside Out, Elsevier Academic Press. 2004. 